\title{
Sinusoidal oscillation of the ankle as a means of evaluating the spastic patient
}

\author{
GERALD L. GOT T LIEB, GYA N C. A G A R A L, A N D \\ R I C H A R D P E N N
}

From the Department of Physiology, College of Health Sciences, Department of Neurosurgery, Rush Medical Center, and College of Engineering, University of Illinois, Chicago, Illinois, USA

SUMMARY Sinusoidally modulated torque was applied to rotate the ankle joint of normal subjects and clinically spastic patients. Measurements were made of the effective joint compliance and of the evoked EMG activity. These procedures provide a well-quantified and reliable measure of muscle tone and hyperreflexia which is well correlated with the patient's clinical status.

The "spastic" patient presents a number of abnormal motor findings such as hyperreflexia, clonus, rigidity, and deficits of voluntary movement. None of these symptoms is well-defined in a quantitative sense, however. This lack of precise quantification creates problems in the consistent evaluation of symptoms over time and necessitates the description of symptoms in qualitative language.

Various measurements can be made in spastic patients which differ from those made in normal subjects. The ratio of the amplitudes of the Hoffmann reflex to the response to direct stimulation of the motor axons ( $\mathrm{H} / \mathrm{M}$ ratio) (Angel and Hofmann, 1963), the Hoffmann reflex recovery curve (Cooper, 1969), and the tonic vibration reflex (Burke et al., 1972; Hagbarth, 1973) have all been described as being able to distinguish between degrees of pathology and health. These methods have not been widely used in clinical evaluation, perhaps because the parameters being measured are too far removed from the abnormal symptomatology which physicians ordinarily see and of which their patients complain.

Methods of directly measuring the resistance of a joint to passive movement have also been described. Webster (1966) measured the amount of work required to cycle the forearm through a peak-to-peak arc of 100 degrees at $20 \mathrm{deg} / \mathrm{s}$. Bosches et al. (1960), Martinez (1966), and Nashold (1966) have all described similar devices

Address for correspondence and reprint requests: Dr G. L. Gottlieb, Department of Physiology, Rush Medical Center, 1753 West Congress Pky., Chicago, Illinois 60612, USA.

Accepted 9 July 1977 which measure a limb's resisting torque as it is displaced at a slow periodic rate by a motor. Duggan and McLellan (1973) have described a method for measuring arm torque during sinusoidal oscillation while sweeping through a frequency range of $1-5 \mathrm{~Hz}$. They computed the energy required to drive the arm at $2 \mathrm{~Hz}$ to distinguish different clinical grades of rigidity.

In the completely relaxed and co-operative sub-⿳⺈⿴囗十一 ject, a joint will resist movement as a result of $\stackrel{\varpi}{\rightleftharpoons}$ three physical processes. First, the inertia of the limb produces reaction forces which are proportional to acceleration. Second, the viscoelastic properties of the muscle and joint will resist movement in a complex way which is a function of both muscle length and its rate of change. Third, muscle contraction may be evoked reflexly by the movement.

The second property is essentially a mechanical description of muscle tone. Tonus is very strongly dependent upon the level of muscular contraction and consequently, activation of muscle either reflexly or voluntarily will have profound effects on it.

The total of all these effects may be characterised by a joint compliance. Compliance is defined as the ratio of joint rotation to applied torque as both change through some interval. A joint with high compliance, such as the normal, relaxed limb, moves easily while the compliance of a tense or rigid limb is low. An important parameter of any compliance measurement is the speed at which the joint is rotated. The magnitude and duration of reflex responses to joint displacement are ex- 
tremely sensitive to the rate of displacement. Hyperexcitability of rate sensitive reflexes is, in fact, one of the apparent problems of the spastic patient. Therefore, compliance should be measured with a range of different rates of displacement.

A number of different methodologies have been used extensively in the fields of engineering for compliance measurement (see Gottlieb and Agarwal, 1977b, for a discussion of different methodologies in this context). One particularly simple one is to apply sinusoidal torques to the joint, and to measure the resulting joint rotation.

In such a measurement, torque $(\tau)$ is described by

$$
\tau=\tau_{\mathrm{b}}+\tau_{\mathrm{p}} \sin (2 \pi \mathrm{ft})
$$

where $\tau_{\mathrm{b}}$ represents a DC bias component, $\tau_{\mathrm{p}}$ is the peak value of applied sinusoidal torque, $f$ is the frequency of oscillation in $\mathrm{Hz}$, and $\mathrm{t}$ is time.

The resulting angular displacement will be

$$
\theta=\theta_{\text {ref }}+\theta_{\mathrm{p}} \sin (2 \pi \mathrm{ft}+\varnothing)+\text { nonlinear terms }
$$

In this equation, $\theta_{\text {res }}$ is a reference angle about which the foot oscillates (which will depend primarily on nonphysiological conditions of the apparatus and whether the subject is asked to contract the leg muscles), $\theta_{\mathrm{p}}$ is the peak sinusoidal component of the resulting angular oscillation, $\phi$ is the phase angle between the applied sinusoidal torque and the resulting rotation, and 'nonlinear terms' refers to any components of the resulting rotation which are not at the same frequency as the driving torque.

From equations 1 and 2, we can define joint compliance $\mathrm{C}$ as

$$
\mathrm{C}(\mathrm{f})=\theta_{\mathrm{p}} / \tau_{\mathrm{p}}
$$

where $\mathrm{C}$ is now a function of the input frequency. In a linear system, one can determine the response to a complex stimulus by considering that stimulus as composed of a sum of simpler stimuli, determine the individual responses of the system to each, and sum the responses. In such a system, the nonlinear term included in equation 2 will be zero. We can make no assumptions about whether that will be true in either the spastic or the normal limb. In fact, such terms might provide a separate index for evaluation, in the time domain if not the frequency, of abnormal function.

In evaluating patients who are potential candidates for implantation of cerebellar stimulators (Cooper et al., 1976) we have measured the compliance of the ankle joint in 14 patients classified as having spastic symptoms from a number of causes. We have also made similar measurements in at least as many normal subjects. The following is a report of our finding on the effects of sinu- soidal oscillation of the ankle joint and how they differ between our two subject populations.

\section{Methods}

Our subjects would sit upright in a chair of adjustable height with the thigh horizontal and the knee flexed about $30^{\circ}$. The foot was firmly strapped to a footplate (Fig. 1) which could rotate freely about

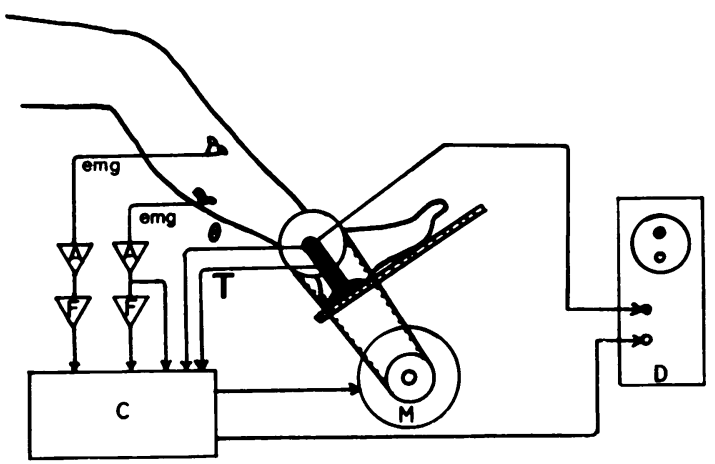

Fig. 1 Experimental motor driven footplate. The computer $(C)$ records the amplified $(A)$, filtered $(F)$ EMGs as well as the angle $(\theta)$ and applied torque $(\tau)$. It drives the torque motor $(M)$. Display oscilloscope (D) shows the subject a signal indicative of foot angle.

a horizontal axis through the medial maleolus. With the subjects as fully relaxed as their condition would allow, the angle of the footplate was adjusted to be perpendicular to the tibia. This was done to compensate for gravitational and other passive torques and for tonic, involuntary contraction, by applying a biasing torque, with a DC torque motor. (In some patients, tonic plantarflexion made this impossible). Subjects were usually given a visual indication of their actual foot angle on an oscilloscope so that inadvertent movements could be noticed and corrected.

The footplate was attached by a belt and pulley system to a DC torque motor (Inertial Motors model 06-024) driven by signals from a digital computer (General Automation, SPC 16/65). The computer generated sinusoidal signals of specified frequencies and recorded foot angle and torque (at 250 samples/second) and electromyograms (at 500 samples/second) measured with pairs of disc surface electrodes on the bellies of the soleus and anterior tibial muscles. The electromyograms were full wave rectified and smoothed with a 10 millisecond filter before recording.

Subjects were instructed to relax while the com- 
puter applied sinusoidal torques for intervals of 11s. Twelve frequencies between 3 and $12 \mathrm{~Hz}$ were used routinely. In some cases additional measurements at frequencies between one and 25 $\mathrm{Hz}$ were also made. Fourier coefficients at each driving frequency were computed for the angle and torque, their ratio being the compliance defined by equation 3 . The average angle and EMG waveforms were also computed (see Agarwal and Gottlieb, 1977a for details).

\section{Results}

To recognise the abnormalities seen in the spastic patients, we must describe briefly the findings in normal subjects. These have been discussed at greater length elsewhere (Agarwal and Gottlieb, 1977a; Gottlieb and Agarwal 1977a).

Figure 2 shows averaged angular and EMG waveforms in a normal subject at different fre-

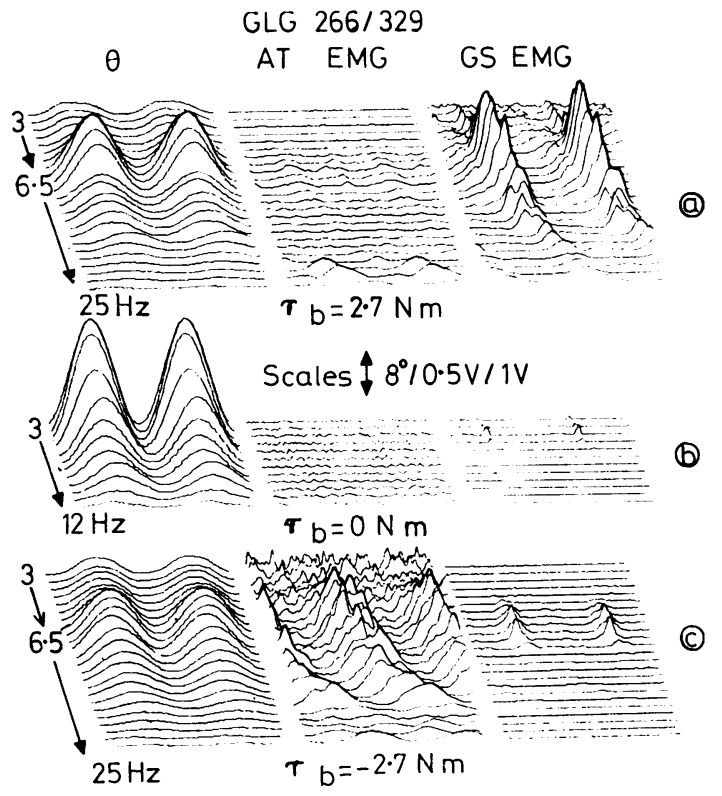

Fig. 2 Averaged foot angle and EMGs from the anterior tibial $(A T)$ and soleus $(G S)$ muscles at different frequencies and levels of bias torque. Positive $\tau_{b}$ indicates that the motor was biased to dorsiflex the foot, requiring tonic soleus contraction to maintain a foot angle centred at the neutral position. In all angle records, up is dorsal. $(a) \tau_{b}=2.7 \mathrm{~N} \mathrm{~m}$. (b) $\tau_{b}=0 \mathrm{~N} \mathrm{~m}$, (c) $\tau_{b}=-2.7 \mathrm{~N} \mathrm{~m}$. Frequencies for $(a)$ and $(c)$ are (3), (3.5), (4), (4.5), (5), (5.5), (6), 6.25, (6.5), 6.75, (7), 7.5, (8), 8.5, 9, 9.5, (10), 10.87, (12), $13.8,15.625,17.8,20$, and $25 \mathrm{~Hz}$. For (b) only those frequencies which are in parentheses were used. quencies of oscillation under three different levels of bias torque $\left(\tau_{1}\right)$. Each horizontal record represents an average of 10 seconds at a single frequency. From foreground to background, records are plotted in order of descending frequency.

Part (b) of Fig. 2 shows twelve frequencies from 3-12 $\mathrm{Hz}$ with zero $\tau_{1}$, and the subject completely relaxed.The peak amplitude of oscillation occurs at $4 \mathrm{~Hz}$. At the largest angular excursions, the EMGs show slight synchronous activation of the soleus muscle. The latency of that activity with respect to the start of the dorsiflexing phase of the cycle-that is, from the point of maximum plantarflexion-is $40 \mathrm{~ms}$.

When the subject opposes a biasing torque of $2.7 \mathrm{~N} \mathrm{~m}$ (Fig. 2a) the oscillation and EMGs change. Peak oscillation occurs at $6.5 \mathrm{~Hz}$, and the soleus EMG shows rhythmic activity which is synchronised with the dorsiflexing phase of stretch. Slight activity can also be seen in the anterior tibial EMG. Near $6 \mathrm{~Hz}$, it is both coactivated with the soleus and also synchronised with the plantar-going phase of the cycle, while at $20 \mathrm{~Hz}$ it is only synchronised with plantarflexion.

Complementary results are seen in Fig. 2c when the bias torque requires a tonic dorsiflexion of $2.7 \mathrm{~N} \mathrm{~m}$. Anterior tibial EMGs are not as well synchronised as were the soleus EMGs of part(a). In spite of voluntary activation of the antagonist of soleus, there is still significantly more soleus EMG activity than when the leg is relaxed.

Figures $3 \mathrm{a}$ and $\mathrm{b}$ show the compliance and phase $\frac{0}{2} \overrightarrow{0}$ as defined by equations 2 and 3 for the relaxed $(X)$ and plantar biased ( $\square$ ) cases. All the above results are reproducible in a normal subject from day to day and between left and right limbs.

Figures 4 and 5 show data from a 24 year old male who had suffered a fracture dislocation of C6-7 vertebrae 12 months previously. Examination of the lower extremities showed hyperactive deep tendon reflexes with ankle clonus and Babinski signs, decreased vibration and position sense, and poor cutaneous sensation. $\mathrm{He}$ was able to walk with crutches.

When oscillation was applied with the subject relaxed, the tonically active EMG of tibialis anterior (AT) was modulated by stretch at all frequencies between 3 and $12 \mathrm{~Hz}$. The timing of this was such that the AT EMG activity appeared to be evoked by dorsiflexion. The AT EMG was maximally active (determined by integrating its area and dividing by the period of oscillation) at 6 and $6.5 \mathrm{~Hz}$. The EMG of gastrocnemius-soleus (GS) showed less evidence of stretch modulation until about $6 \mathrm{~Hz}$. Peak GS activity was also at 6 to $6.5 \mathrm{~Hz}$. 


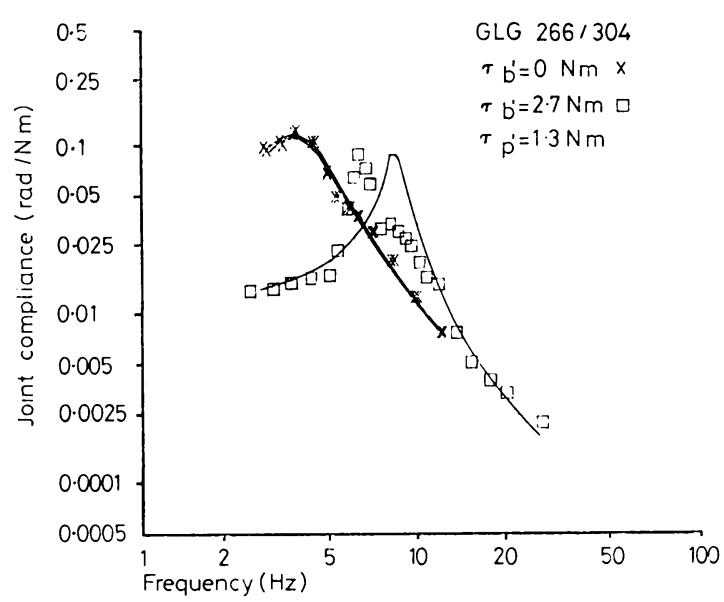

(a)

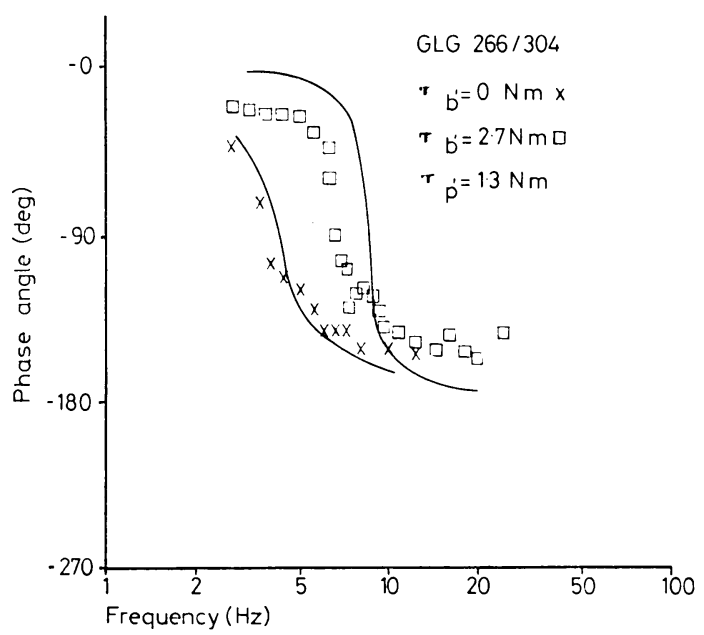

(b)

Fig. 3 Normal compliance with the subject relaxed $(X)$ and exerting a tonic plantar flexion $(\square)$ of 2.7 $N \mathrm{~m}$. Data correspond to parts $(b)$ and $(a)$ of Figure 2. $b=$ phase of the joint angle with respect to the applied torque. Solid lines are from the model of equation 5. (See text).

Asking the subject to plantarflex voluntarily against a motor bias torque of $1.3 \mathrm{~N} \mathrm{~m}$ produced a decrease in AT EMG activity and an increase in GS EMG activity. The low frequency compliance diminished slightly and a very sharp resonance appeared around $6.25 \mathrm{~Hz}$. The maximum GS EMG activity was between $5.5 \mathrm{~Hz}$ and $6.25 \mathrm{~Hz}$.

Figure 6a shows five seconds of oscillation at $7.5 \mathrm{~Hz}$. Note that big and small rotation cycles alternate. Both AT and GS EMGs show activity after the big cycle dorsiflexions with latencies from maximum plantarflexion being about $30 \mathrm{~ms}$.

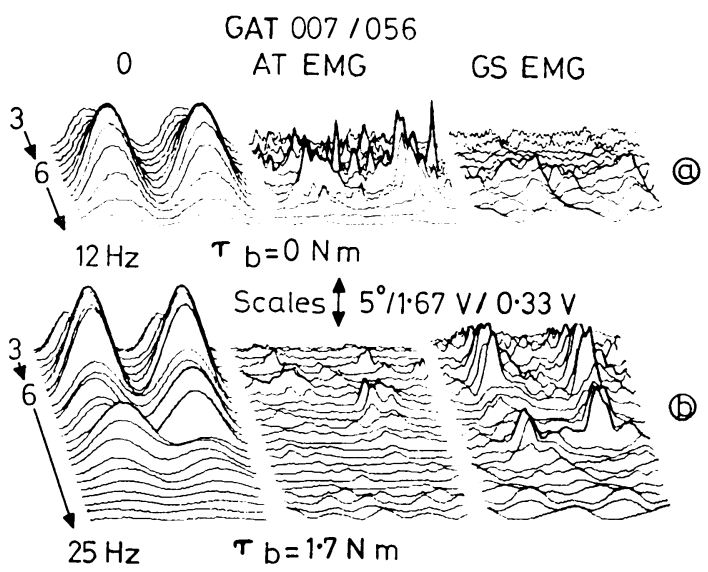

Fig. 4 Average angle and EMG records of a spastic subject under $(a)$ relaxed and $(b)$ plantar biased conditions. See Fig. 2 caption for frequencies used.

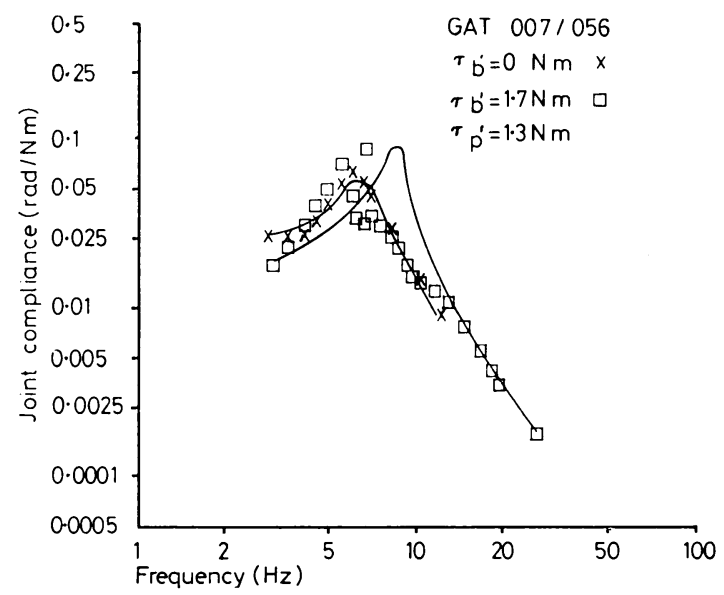

Fig. 5 Compliance of the ankle of subject from Fig. 4. $\times=$ relaxed leg. $\square=$ tonic plantar contraction. Solid lines are from the model of equation 5.

Figure $6 \mathrm{~b}$ shows approximately eight seconds of oscillation at $6.25 \mathrm{~Hz}$. The rotation of the foot and the GS EMG bursts are pretty much the same from cycle to cycle. The latency of the GS EMG bursts from the most plantarflexed point in the cycle is about $30 \mathrm{~ms}$. The unusual feature of this record is that AT EMG activity is highly asymmetric. After the first four seconds, AT EMG activity bursts every other cycle while GS activity continues to occur every cycle.

Figures 7 and 8 show data from a 24 year old male cerebral palsy patient. He showed rigidity in both lower limbs, the right limb being considerably 
(a)

$$
\text { GAT } 0.47 \quad 7.5 \mathrm{~Hz} \quad \tau_{b}=1.7 \mathrm{Nm}
$$

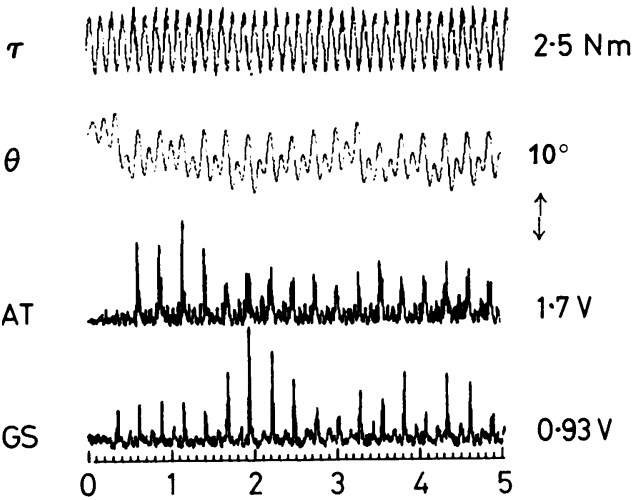

(s) (b)

$$
\text { GAT } 055 \quad 6.25 \mathrm{~Hz} \quad \tau_{\mathrm{b}}=1.7 \mathrm{Nm}
$$
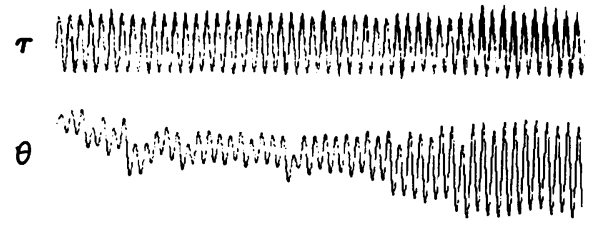

$\uparrow$

$2 \cdot 5 \mathrm{Nm}$

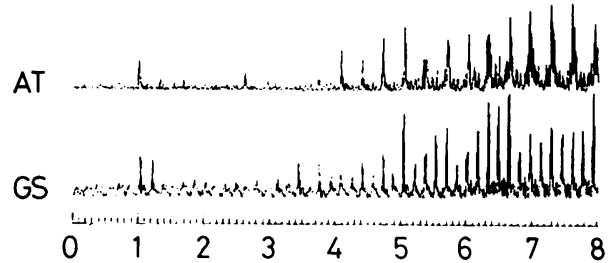

(s)

Fig. 6 Oscillation of the foot of subject from Fig. 5 at (a) $7.5 \mathrm{~Hz}$ and (b) $6.25 \mathrm{~Hz}$. Oscillation at $7.5 \mathrm{~Hz}$ shows the common phenomenon of alternate large and small cycles and EMG activity every other cycle. Oscillation at $6.25 \mathrm{~Hz}$ shows symmetric oscillation with GS EMG bursting every cycle and AT EMG bursting every other cycle.
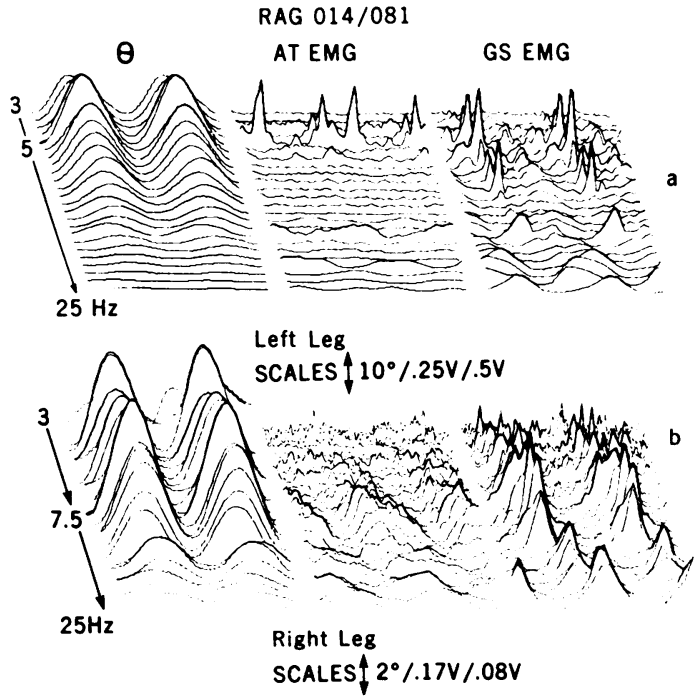

Fig. 7 Average angle and EMG records of left $(a)$ and right $(b)$ ankles of a cerebral palsy patient. Both legs relaxed.

stiffer than the left. Ankle jerk reflexes were difficult to elicit because of the hypertonus nor could a Babinski sign be demonstrated. Figure 7 shows average waveforms in both right and left legs with the subject relaxed and no bias torque applied. Figure 8 shows the corresponding compliance curves.

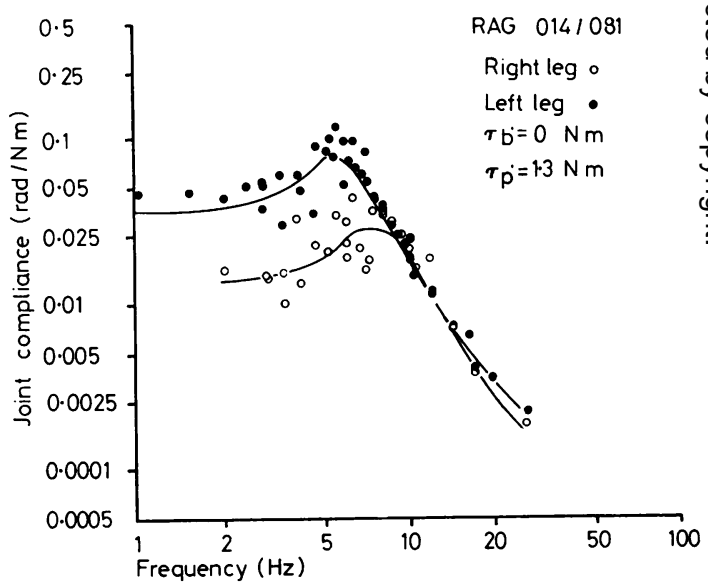

Fig. 8 Compliance of right (O) and left $(\bullet)$ ankles of subject from Fig. 7. Both legs relaxed.

\section{Discussion}

The consistent finding in all clinically spastic patients we have seen thus far is that their relaxed ankle compliance, measured at frequencies below $4 \mathrm{~Hz}$, is less than normal by a factor which is consistent with the degree of rigidity found clinically. This increased stiffness is not a consequence of phasic contraction of the muscle acting about the 
ankle joint. The evidence for this is that examination of the average EMG records below $4 \mathrm{~Hz}$ fails to reveal any evidence of stretch modulation of the EMG waveform.

A second consistently observed finding is the presence of a resonance in the $6-8 \mathrm{~Hz}$ region of the relaxed compliance curve. In this frequency range, stretch modulation of the EMG waveform is quite evident, often showing coactivation of agonist and antagonist muscles after the start of the dorsiflexing phase of the cycle.

The presence of this resonance can be explained in terms of the known properties of muscle and the spinal stretch reflex. The twitch evoked by a rapid stretch of the triceps surae develops its peak tension at a latency between $125 \mathrm{~ms}$ and $167 \mathrm{~ms}$, times which correspond to the periods of oscillation at 8 and $6 \mathrm{~Hz}$. Thus, the dorsal-going phase of each cycle may evoke a stretch-reflex contraction with tension developing during the plantargoing phase of the motor's cycle.The torque motor and the muscle would then act together to increase the amplitude of oscillation.

In these respects then, the relaxed spastic subject responds to oscillation just as would a normal subject who exerts a slight voluntary plantarflexion. Low frequency compliance is reduced. Above $4 \mathrm{~Hz}$ there is rhythmic modulation of soleus EMG activity and there is a resonance in the compliance between 6 and $8 \mathrm{~Hz}$. There seems to be considerably more coactivation of the anterior tibial and the soleus muscles than is seen in either the relaxed or the voluntarily plantarflexing normal subject.

The compliance data presented in Figs. 3, 5, and 8 lend themselves to a simple and useful procedure for quantification. The data can be approximated by a linear mechanical system consisting of a moment of inertia $(\mathrm{J})$, a viscous element (B), and an elastic element (K).

Such a system is described by the linear differential equation

$$
\tau=\mathbf{J} \frac{\mathrm{d}^{2} \theta}{\mathrm{dt}^{2}}+\mathbf{B} \frac{\mathrm{d} \theta}{\mathrm{dt}}+\mathrm{K} \theta
$$

where $\tau$ and $\theta$ are the torque and angular rotation. Taking the Laplace transform allows us to define a complex compliance as

$$
\mathrm{C}(\mathrm{S})=\frac{\theta}{\tau}=\frac{1}{\mathrm{JS}^{2}+\mathrm{BS}+\mathrm{K}}
$$

where $\mathrm{S}$ is the complex frequency. The solid lines in Figs. 3, 5, and 8 represent the 'best' fit of equation 5 to the data. The computed values for $\mathbf{J}, \mathbf{B}$, and $\mathrm{K}$ are shown in the table.
The parameter $\mathrm{K}$ is the 'spring constant' of the muscle which characterises its elastic behaviour at low frequencies. We have shown elsewhere (Agarwal and Gottlieb, 1977b) that this parameter varies linearly with muscle tension in normal subjects and it provides a simple measure of muscle tone. The dependence of muscle compliance on its level of activation is well-established (Wilkie, 1950), and is presumably based on the fundamental lengthtension properties of the muscle sarcomere. The value of $\mathrm{K}$ is determined by the low frequency compliance of the muscle and appears to be characterised adequately by measurements at 2-3 Hz.

The value of the moment of inertia $\mathbf{J}$ is determined by the high frequency compliance terms. The inertia is constant in an individual subject but the estimates from the data vary for two reasons. The curve fitting procedure which establishes the best fit is one source of variance. Of greater import is that the true compliance of the joint varies in a far more complex manner than can be explained by a three element mechanical model, and the parameters are influenced by more complex processes (such as reflex effects) than just the simple mechanical properties they represent.

The viscous damping parameter $\mathbf{B}$ is determined largely by the midfrequency data near resonance. It is consequently very sensitive to the definition of 'best' fit. In the experiments with the relaxed limb shown previously, all three parameters can be chosen to give a good fit by minimising the criterion

$$
\text { error }=\sum_{\mathbf{f}}\{\log (\mathrm{C} \text { measured } / \mathrm{C} \text { model })\}^{2}
$$

which provides equal weighting to data at all frequencies. In the two cases shown where the subjects contracted voluntarily against a bias torque, the fit from this criterion is far less satisfying. The solid curves for these cases were generated by selecting $\mathrm{K}$ and $\mathrm{J}$ to give good fits at high and low frequencies and then selecting $B$ to create a resonant peak of equal height to the measured peak.

In the linear model, the resonant frequency is equal to $\sqrt{\mathrm{K} / \mathrm{J}} / 2 \pi \mathrm{Hz}$. Clearly, the sharp resonances observed in the compliance cannot be explained in tcrms of such a simple "mass-spring" phenomenon because they occur at too low a frequency. From the EMG data, it is reasonable to infer that stretch reflex mechanisms are making a significant con:ribution in this region of the spectrum and account for the difference between the model and the limb. 
Whether those reflex processes are essentially spinal, however, is not immediately evident. Figure 6 shows two phenomena. In Fig. 6a, stretch cycles are alternately big and small, only the big ones evoking a reflex EMG burst. This behaviour is often seen in normal subjects in the $8-12 \mathrm{~Hz}$ range, and can be accounted for by the same physiological properties that produce the resonance. (At about twice the resonant frequency, the big dorsiflexing phase elicits a reflex plantar contraction which opposes the dorsiflexing phase in the cycle which immediately follows it.)

The behaviour in Fig. 6b, however, has never been observed in a normal subject. Here we see GS activity evoked by every cycle of stretch while AT activity is predominantly every other cycle. The angular oscillation remains essentially symmetric from cycle to cycle. It is difficult to explain such a response solely in terms of peripheral reflex mechanisms. In the records of Fig. 6a, the nonlinear terms in equation 2 may actually contain most of the signal energy.

It is noteworthy that the modulation of the EMGs seen in Fig. 7 provides evidence of hyperactive phasic stretch reflexes although tendon jerks could not be elicited in this subject. The Table summarises the data from five normal adult subjects and four adult patients. The rest of our patients were children. The parameters were estimated using the criteria of equation 6 except those in parentheses. Values in parentheses were computed using low frequency measurements alone to estimate $\mathrm{K}$, and high frequency measurements alone to estimate $\mathbf{J}$.

The Table includes the mean error of the model fit-the final value of equation 6 divided by the number of frequencies used. In those cases where there was a strong resonance in the measured compliance, this error is much larger than when there was no resonance.

These data suggest that the best second order approximation of the relaxed normal ankle has a resonant frequency below $5 \mathrm{~Hz}$, a damping factor greater than 0.2 and a spring constant of less than $25 \mathrm{~N} \mathrm{~m} /$ radian. The error criterion is small indicating that the quality of the approximation is good. Even when all these criteria are not met, the differences between normal relaxed and normal contracted parameters are dramatically different.

In patients with clinical spasticity the resonant frequency tends to be above $5 \mathrm{~Hz}$ and the spring constant greater than $25 \mathrm{~N} \mathrm{~m} /$ radian. In such cases, the error is much greater than before, and, therefore, the parameter estimates have less meaning. Using low frequency data alone is, in fact, a more satisfying method of measuring the elastic stiffness of the joint - this would be equivalent to the method of Duggan and McLellan (1973). These patients also show qualitatively similar responses whether relaxed or contracted.

It is clear, however, that we must interpret ab solute numbers with care. The data from PJP026 a cerebral palsy patient, show the smallest value of elastic stiffness and, in fact, on the date thoseo measurements were made the patient was not rigice although other spastic symptoms were present $\delta$ One week later (PJP125) his clinical picture had changed and this change is clearly reflected in the measurements.

We must also note that although we use a simple linear approximation describable by a transfer function (equation 5), we do so only to provide a concise (but very crude) means of describing the response with a few numerical values. The measurements themselves each describe the com-

Table Summary of data from five normal adult subjects and four adult spastic patients

\begin{tabular}{|c|c|c|c|c|c|c|}
\hline & $\begin{array}{l}J \\
N m s^{2} / \text { radian }\end{array}$ & $\begin{array}{l}B \\
N m \text { s/radian }\end{array}$ & $\begin{array}{l}K \\
N m / \text { radian }\end{array}$ & $\begin{array}{l}E \\
\text { Damping factor }\end{array}$ & $\begin{array}{l}f_{n} \\
H z\end{array}$ & $\begin{array}{l}E \\
\%\end{array}$ \\
\hline \multicolumn{7}{|c|}{ Normal subjects } \\
\hline SMB & 0.0304 & 0.422 & 29.6 & 0.223 & 4.93 & 0.241 \\
\hline GCA & 0.0207 & 0.206 & 22.0 & 0.153 & 5.19 & 0.325 \\
\hline PAG & 0.0334 & 0.496 & 22.9 & 0.283 & 4.14 & 0.025 \\
\hline BWF & 0.0247 & 0.272 & 17.5 & 0.207 & 4.24 & 0.033 \\
\hline GLG-relax & 0.0246 & 0.305 & 17.1 & 0.236 & 4.19 & 0.092 \\
\hline \multirow[t]{2}{*}{ GLG-Bias } & 0.027 & 0.369 & 58.0 & 0.0157 & 7.39 & 2.08 \\
\hline & $(0.0246)$ & (0.190) & (73.0) & $(0.071)$ & (8.67) & (6.25) \\
\hline \multicolumn{7}{|l|}{ Spastic patients } \\
\hline PJP026 & 0.0178 & 0.225 & 11.25 & 0.251 & 4.00 & 0.217 \\
\hline PJP125 & 0.0139 & 0.790 & $16.2(30)$ & 0.831 & 5.43 & 1.050 \\
\hline FWB011 & 0.0210 & 0.391 & 30.52 & 0.224 & 6.06 & 2.05 \\
\hline RAG (Rt. 1) & 0.0243 & 0.648 & 69.0 & 0.250 & 8.47 & 1.24 \\
\hline RAG (L. 1) & 0.0192 & 0.295 & 27.7 & 0.202 & 6.05 & 0.889 \\
\hline GAT001 & 0.0260 & 0.387 & 45.4 & 0.179 & 6.65 & 0.140 \\
\hline \multirow[t]{2}{*}{ GAT-bias } & 0.0226 & 0.486 & 39.4 & 0.257 & 6.64 & 1.06 \\
\hline & $(0.0226)$ & $(0.200)$ & $(57.0)$ & $(0.088)$ & (7.99) & (5.87) \\
\hline
\end{tabular}


pliance of the joint at a single frequency but the group of measurements over the whole frequency spectrum do not describe a realistic transfer function. From sinusoidal measurements one cannot predict the rotation of the ankle to other kinds of torque signals.

The approximation is only useful because it allows us to make consistent comparisons: between subjects, between left and right limbs, and between successive sessions. With this limitation, the method yields precise and stable results which accurately reflect the subjects' clinical states.

\section{Conclusion}

It has been our experience that simple compliance measurements using sinusoidal waveforms provide useful information about muscle tone and phasic reflex excitability. They provide a quantitative measure that is consistent with the patient's clinical status and are obtained without any discomfort.

The methods described here for measuring compliance could be considerably simplified. It is much simpler to build a constant-displacement, sinusoidal drive using small geared AC motors. It would only be necessary to measure the RMS torque to obtain the compliance.

EMG data, if used, could be qualitatively observed on an oscilloscope. Only five frequencies -for example, 2, 3, 5, 7, $9 \mathrm{~Hz}$-applied for 10 seconds each would be needed to characterise the resonance. Such a system would be extremely simple to build and operate and would provide well-quantified, reproducible information about patient status that is not now conventionally available.

This work was supported in part by NINCDS grant NS00196, NSF grant ENG-7608754, and The Susman Asher Foundation.

\section{References}

Agarwal. G. C., and Gottlieb, G. L. (1977a). Oscillation of the human ankle joint in response to applied sinusoidal torque on the foot. Journal of Physiology, 268, 151-176.
Agarwal, G. C., and Gottlieb, G. L. (1977b). Compliance of the human ankle joint. Journal of Biomechanical Engineering, 99, 166-170.

Angel, R. W., and Hofmann, W. W. (1963). The H reflex in normal, spastic and rigid subjects. Archives of Neurology (Chicago), 8, 591-596.

Boshes, B., Wachs, H., Brumlik, J., Mier, M. and Petrovick M. (1960). Studies in tone, tremor and speech in normal persons and parkinson patients. I Methodology. Neurology (Minneapolis), 10, 805-813.

Burke, D., Andrews. C. J., and Lance, J. W. (1972). Tonic vibration reflex in spasticity, Parkinson's disease and normal subjects. Journal of Neurology. Neurosurgery, and Psychiatry, 35, 477-486.

Cooper, I. S. (1969). Involuntary Movement Disorders. Hoeber: New York.

Cooper, I. S., Riklan, M., Amin, I., Waltz, J. and Cullinan, T. (1976). Chronic cerebellar stimulation in cerebral palsy. Neurology (Minneapolis), 26, 744753.

Duggan, T. C., and McLellan, D. L. (1973). Measurement of muscle tone: a method suitable for clinical use. Electroencephalography and Clinical Neurophysiology, 35, 654-658.

Gottlieb, G. L., and Agarwal, G. C. (1977a). Physiological clonus in man. Experimental Neurology, 54, 616-621.

Gottlieb, G. L., and Agarwal, G. C. (1977b). Two methods of measuring the dynamic behaviour of the stretch reflex in man. Proceedings of the 1977 San Diego Biomedical Symposium. Volume 16. pp. 369374. Edited by J. I. Martin. Academic Press: New York.

Hagbarth, K-E. (1973). The effect of muscle vibration in normal man and in patients with motor disorders. In New Developments in Electromyography and Clinical Neurophysiology, Volume 3. Edited by J. F. Desmedt. Karger: Basel.

Martinez, N. (1966). Measurement of rigidity with a strain gauge myokinetograph. Journal of Neurosurgery, 24, 315-316.

Nashold, B. S. (1966). An electronic method of measuring and recording resistance to passive muscle stretch. Journal of Neurosurgery, 24, 310-314.

Webster, D. D. (1966). Rigidity in extrapyramidal disease. Journal of Neurosurgery. 24, 299-307.

Wilkie, D. R. (1950). The relation between force and velocity in human muscle. Journal of Physiology, 110, 249-280. 